\title{
Post-Secondary Student Stress: A Qualitative Descriptive Study (Preprint)
}

Brooke Linden, $\mathrm{PhD}$ and Heather Stuart, $\mathrm{PhD}$

Health Services and Policy Research Institute, Queen’s University, Kingston, ON, Canada

March 2020

\begin{abstract}
Purpose: Excessive stress among post-secondary students has been routinely linked to negative academic and mental health outcomes. The purpose of this qualitative descriptive study was to invite students to identify salient sources of stress within the post-secondary setting in order to facilitate improved measurement of student stress moving forward.

Methods: Focus group interviews were conducted with students from a variety of levels and areas of study. Data was thematically coded into major themes and sub themes, with direct quotes extracted for support.

Results: Five major themes of stress were identified, including academics, the learning environment, campus culture, interpersonal, and personal stressors, revealing a multidimensional concept of student stress.

Conclusions: Underlying challenges were revealed, included time management, fear of failure, mental health literacy and education among staff and faculty, and campus inclusivity. The implications of these findings are discussed in the context of existing literature and directions for future research are identified.
\end{abstract}

Keywords: stress, post-secondary, mental health, focus groups, qualitative research 


\section{Introduction}

Excessive stress among post-secondary students is associated with a number of negative health outcomes, including poor mental health (Eisenberg, Gollust, Golberstein, \& Hefner, 2007; Gollust, Eisenberg, \& Golberstein, 2008; Patel, Flisher, Hetrick, \& McGorry, 2007) and declining academic performance (American College Health Association, 2016). The majority of Canadian post-secondary students fall within the 18 to 25 year age bracket, a period referred to as "emerging adulthood" (Arnett, 2000). Emerging adulthood is comprised of increased independence and responsibility compounded by a lack of role permanence, creating a vulnerable period where individuals may be more susceptible to the effects of chronic stress. Indeed, emerging adults fall within the age group identified as the most likely to self-report symptoms of mental illness as well as an unmet need for mental health care (Pearson, Janz, \& Ali, 2013; Sunderland \& Findlay, 2013). Additionally, the most recent edition of the National College Health Assessment II survey revealed a substantial prevalence of both stress and common mental illnesses, such as anxiety and depression, among Canadian post-secondary students (American College Health Association, 2019). The same survey revealed severe psychological distress to be evident among a proportion of surveyed students, demonstrated by suicidal ideation $(13 \%)$, self-harm $(8.7 \%)$, and previous suicide attempts $(2.1 \%)$. These findings are particularly concerning given that suicide is the second leading cause of death among young Canadians (Navaneelan, 2012).

Existing literature has agreed upon several common areas of stress for students (e.g., particularly those related to academics). However, as our world becomes more complicated and multidimensional, students potentially face more stressors today than ever before, many of which exist outside of the academic sphere but are still specific to the social context of being a student. With the advent of things like social media, the changing cost and value of education - with more than half of Canadians holding post-secondary qualifications in 2016 (Statistics Canada, 2017) and the average student debt incurred from a bachelor's program at an all-time high (Walsh, 2018) - and the changing economic landscape in Canada, today's students face stressors unheard of by previous generations. Although a growing body of evidence has acknowledged the negative impacts these stressors can have on post-secondary students' mental health and wellbeing, there remains a lack of in-depth research into the specific sources of this stress. It is important to move beyond commonly held beliefs about the sources of stress within the post-secondary setting, and invite students to share their experiences in order to develop a more holistic understanding of student stress. An improved evaluation of the sources of post-secondary student stress is warranted in order to more effectively target upstream mental health promotion and mental illness prevention services on campuses.

Existing instruments used to measure stress among post-secondary students are imperfect for a number of reasons. Few have involved a diverse sample of students in the development process (e.g., engaging only students in a particular year, level, or program of study), while others have been developed too narrowly (e.g., items developed based solely on literature, little consideration for student input) or too broadly (e.g., including stress-related items irrelevant to the post-secondary experience). The purpose of this qualitative descriptive study was to engage current students in detailed discussions to develop an inventory of stressors specific to the role of "student" in order to facilitate improved measurement of student stress moving forward. This study formed the initial work in support of 
the development of the Post-Secondary Student Stressors Index, a new, holistic instrument designed to assess the multidimensional sources of student stress (Linden \& Stuart, 2019).

\section{Methods}

\section{Design}

We used a relativist ontology to understand the social contexts and stressful experiences of postsecondary students within the university setting. Qualitative descriptive designs provide a rich description of a phenomenon of interest, emphasizing the exploration of ascribed meaning according to individuals from within the natural setting (Bradshaw, Atkinson, \& Doody, 2017). Because the goal of this study was to invite students to identify salient sources of stress within the post-secondary setting, a qualitative describe design was suitable. Ethical approval was granted for this study by the [blinded] University's Health Sciences and Affiliated Teaching hospitals Research Ethics Board (HSREB).

\section{Participants and Eligibility}

Purposive sampling was used to recruit students to participate in focus group interviews. Students belonged to multiple groups within the postsecondary community. Recruitment ads were posted at multiple sites across campus in areas where students experiencing stress may seek support (e.g., student wellness centre, peer support office, etc.). The goal was to speak to "like" groups of students (e.g., undergraduates, graduate students, international students, etc.) in order to take advantage of common experiences of stress and promote idea sharing in conversations. Through a QR code or URL, advertisements linked prospective participants to a brief registration survey, which requested brief contact and demographic information (sex, level of study, student status, year of study, area of study). From this registration data, six groups were formed: two undergraduate groups, two graduate student groups, one group with male students only, and one group with medical students only. The only eligibility criteria were that 1) students be enrolled during the Fall 2018 semester at [blinded] University, 2) were willing to share their experiences of stress, and 3) and be able to communicate in English. A total of 17 students participated in the study.

\section{Data Collection}

Semi-structured, face-to-face focus groups were facilitated by the principle investigator (BL). Groups ranged in size from 2-5 participants, were approximately 1.5 hours in length, and were audio recorded and transcribed. A loose interview agenda was utilized to offer direction to participants. Participants were asked to share their experiences regarding stressors in the postsecondary setting (e.g., What causes you stress as a student?) to support the development of a new instrument designed to better evaluate postsecondary student stress. Participants were also asked to provide context around each item (e.g., Why is this stressful?).

\section{Data Analysis}

Data were coded using applied thematic analysis, an iterative process where the data are analyzed for patterns in participants' responses and organized into thematic categories, while "codes" are applied to indicate the semantic boundaries of each theme (Guest, MacQueen, \& Namey, 2012). For example, responses about stressors related to academics were categorized under the "academic" theme, while codes such as "exams and tests" and "grades" were applied to subcategories of stressors. All coding was completed by the principle investigator (BL) using NVivo (Version 12.1). The data analysis generated five overarching themes of student stress, with supporting quotes extracted to provide contextual support for each theme. 


\section{Results}

Focus group participants $(n=17)$ were largely female $(65 \%)$ and studied in a variety of departments and years of study (Table 1). Through focus group interviews, participants added 12 additional items to the initial pool, resulting in an inventory of 80 stressors in total. Five themes of student stress were derived from focus group data: 1) academics, 2) learning environment, 3) campus culture, 4) interpersonal, and 5) personal stressors. Stressors within each theme were coded into 2-4 subcategories within each theme, with a total of 68 individual stressors identified by participants (Table 2).

Table 1. Demographics for Focus Group Participants

\begin{tabular}{lcc}
\hline Variable & Percent & Freq \\
\hline Sex & & \\
\hline Female & $65.0 \%$ & 11 \\
$\quad$ Male & $35.0 \%$ & 6 \\
\hline Level of Study & & \\
$\quad$ Undergraduate & $53.0 \%$ & 9 \\
Professional & $23.0 \%$ & 4 \\
Masters & $18.0 \%$ & 3 \\
Doctorate & $6.0 \%$ & 1 \\
\hline Area of Study & & \\
\hline Business & $29.0 \%$ & 5 \\
Medicine & $23.0 \%$ & 4 \\
Science/Nursing & $18.0 \%$ & 3 \\
Education & $18.0 \%$ & 3 \\
Arts & $12.0 \%$ & 2 \\
\hline
\end{tabular}

\section{Academics}

Perhaps unsurprisingly, the largest number of stressors belonged to the "academics" theme. Sub-categories included: exams and grades; managing the academic workload; class requirements; and meeting the requirements of one's program.

Exams and Grades. Almost all students reported feeling pressured to achieve and maintain high grades. Exams were frequently acknowledged as a source of stress, from preparing for and writing exams, to worrying about how heavily one mark might impact a student's overall performance in a course. One student explained:

"You start to think about what you need to get an A, start thinking about how much messing up one question is going to affect your grade [...] If I can't get this question, then I won't get this grade in the course, and it just spirals."

Table 2. Themes and Sub-Themes Identified

\begin{tabular}{ll}
\hline Theme & Subcategories \\
\hline Academics & Exams and Grades \\
& Academic Workload \\
& Class Structure \\
& Program Requirements \\
\hline Learning & Interactions with Staff and \\
Environment & Faculty \\
& Interactions with Teaching \\
& Assistants \\
& Relationship with Supervisor \\
& Physical Learning Environment \\
\hline Campus & Social Pressures \\
Culture & Discrimination \\
& Competitive Atmosphere \\
& Adjustment to School \\
\hline Interpersonal & Relationship Management \\
& Meeting Expectations \\
\hline Personal & Self-care and Health \\
& Work/Life Balance \\
& Financial Stressors \\
& Concern for the Future \\
\hline
\end{tabular}

Notably, many students emphasized that much of the stress they associate with exams stems not from the simple necessity of having to write and perform well on an exam, but rather from having to manage crowded exam schedules. On being faced with several back-to-back exams in one week, one student remarked:

"I didn't prepare for exams - I passed. That's all I needed. My grades were terrible that year. That was the one semester I was not very proud of." 
While maintaining a high grade point average was important to many students, several emphasized the stress associated with being graded on a curve, relative to their peers' performance. Participants noted that this experience was particularly stressful in courses designed to build on each other (i.e., in an introductory statistics course meant to build on a more challenging future statistics course). On bell curved grades, one student noted:

"If everyone gets bad marks, we all get bumped up, but nobody learns."

Academic Workload. Almost every student expressed having experienced significant stress in attempting to manage their academic workload. Of all of the stressors reviewed during this study, those within this domain were consistently the most commonly reported across student groups, with both graduate and undergraduate students noting the continuing challenge of time and workload management. One student (now beginning to study at the graduate level) remarked:

"I spent the last four years just trying to keep my head above water."

Students remarked on both the quantity of deliverables expected of them, as well as regularly having multiple assignments and other evaluations due within a short time frame. On deadlines "piling up", one participant shared:

"All the deadlines are always so close together. I just wish professors would communicate [with each other]."

Students linked their concern surrounding meeting deadlines to grade-related stress, noting the potential domino effect of poor time management:
"If you don't meet your deadlines, you don't pass your course, and you don't get your degree, and then you can't get a job, and you're unsuccessful..."

Class Structure. Students also identified certain components of the class structure as sources of stress. While several students identified having to participate in class as a source of stress, many noted that a large proportion of that stress stemmed from worrying about their peers' perceptions of their contributions. One student explained:

"In some classes, we get participation marks for asking questions in class. But it's not just about asking questions; it's about asking 'good' questions. You're always worried that your peers are going to make fun of you for asking a stupid question."

Another source of academic stress for students stemmed from having to participate in group work to satisfy the requirements for a course, again linking this stressor to grade-related stress. Students emphasized the challenges associated with being forced to rely on other group members to achieve an acceptable grade. One student noted:

"If [your group members] aren't engaged with what's going on, it's hard to do your best. Are people going to get things done on time? Are they going to pull their weight? [...] To have your grade hinge on someone else is the worst feeling in the world."

Program Requirements. Many students, particularly those studying at the graduate level or in professional programs, identified meeting their program requirements as a major source of stress. One such requirement was the pressure to publish, often felt in graduate and medical school. Students reported feeling as though the need to 
publish academic papers was a "hidden curriculum":

"Sometimes we aren't explicitly told that we need to publish, but it's a hidden curriculum for sure [...] It's not necessary, but there's a lot of pressure to do it."

Finally, meeting program "milestones" (e.g., passing comprehensive exams, submitting a major proposal, completing a dissertation, etc.) was identified as a source of stress and a source of comparison to others. The stressor looked different depending on students' level and area of study. Medical students and students in professional programs emphasized the stress associated with performing well in major work placements (e.g., clerkship or practicum). For undergraduate students, milestones varied by program, while every graduate student identified their required coursework, thesis proposal, and defense as stressful milestones with a large impact on their educational and professional future. One student explained:

"Because everyone in the graduate program is so driven [...] you're with this cohort that's very accomplished. Watching them hit milestones can be intimidating when you're a little bit behind. Always comparing yourself to your peers is hard."

\section{Learning Environment}

Stressors belonging to the learning environment domain largely related to interactions with faculty, teaching assistants, and supervisors.

Interactions with Professors. In general, interacting with faculty was a common source of stress for students, particularly at the undergraduate level. Students identified things such as poor communication, unclear expectations, and lack of guidance or understanding from professors as primary sources of stress within the learning environment. One student shared:

"I've had courses where we're taught theory, but the exams are applied problems. I had a professor tell me, "Well, if you wanted to learn how to do it you should have go to college, not university. We teach theory."

Students also identified specific interactions with professors in the classroom as stressful, including asking for clarification on a grade, asking for an assignment to be remarked, asking for an extension, and asking for a formal academic accommodation. One student shared:

"I found sometimes having to ask for an extension I felt incredibly uncomfortable or judged. [...] I felt uncomfortable asking for what I knew I needed."

With respect to academic accommodations, students discussed not only the perceived stigma experienced in interactions with professors, but also an overwhelming level of self-stigma associated with arranging for an accommodation. Several students reported feeling a sense of guilt or failure to meet the expectations of others, or themselves, whether it be a formal academic accommodation approved through the institution, or a more informal request (i.e., an extension).

"I hate asking for accommodations. I have an expectation for myself to meet [deadlines]. I've thought about it, and then been like "Don't you dare!" [...] We need to work on changing that idea that [accommodations are] bad."

Interactions with Teaching Assistants. Students suggested items relating to interactions with teaching assistants (TA) be added to the instrument. Examples of these stressful interactions included having an unhelpful, unqualified, or unprofessional TA. Another 
challenge students identified was associated with TA's often being peers or classmates. Several students acknowledged the lack of professionalism frequently shown by TA's as a source of stress. One student shared:

"[My] TA that would go out drinking with a group of students in the class, and [those students] did very well... but you wonder, are they doing well because they're working hard, or because they're going for drinks with the TA every Friday?"

Relationship with Supervisor. For graduate and professional students, one of the most salient sources of stress within the learning environment stemmed from the relationship with their thesis or placement supervisor. This included stressors such as feeling unable to meet the supervisor's expectations, experiencing a lack of mentoring, or perceiving there to be a lack of recognition. One student shared their experience with "sharing" a supervisor with others, noting:

"I feel like my supervisor hasn't been as hands on with me as she has with other students [...] It nags at me in the back of my mind like, "Does she really want to be supervising me? Am I not good enough?" When there's any disconnect between you and your supervisor, it adds this additional layer to navigate."

Other students spoke about a perceived expectation for misery during graduate school, a sentiment often promoted by supervisors and other faculty members. One student shared:

"There's almost a spirit of initiation in grad school [...] This idea of like, I went through this, so now you have to go through it. You have to 'earn your stripes.",

Another student shared an interaction they experienced after navigating a substantial reduction in mental health as a result of the demands of a doctoral workload:

"She said to me, "It's your PhD - everybody cries. It's normal."

\section{Campus Culture}

Stressors belonging to the campus culture domain were associated with socio-environmental pressures, including social pressures, experiences of discrimination, competition among peers, and adjustment to the post-secondary setting.

Social Pressures. Among male students in particular, social pressures associated with substance use were one of the most commonly reported sources of stress on campus. Many of the experiences related to substance use carried with them an aspect of meeting expectations to perform or behave in a particular way. One student shared:

"You're in a new space, with new people, and [you want] make sure that people like you. If you drink a lot, it means you have fun, you went out in high school, all this stuff... there's so many bigger things that are worth more time than just getting outrageously drunk."

Often, students expressed disdain for engaging in substance use, particularly to excess. However, feelings of obligation and futility were also commonly expressed.

"We've been out the last four nights in a row... I don't want to go out! I don't want to. [...] If you don't show up, people judge you. [...] There's always a reason you have to go out. There's always $\$ 2$ drinks somewhere."

Discrimination. Several students identifying as ethnic minorities acknowledged experiences of racism on campus as a major source of stress. Many students discussed the racism they 
experienced as being comprised of frequent, subtle micro-aggressions, rather than overt or explicit acts of racism:

"It's not the overt kind of racism [you might think of], but it's the small micro-aggressions like, moving away from [me], not wanting to sit beside me, not saying hi."

Another student discussed feeling as though one's value as a peer and colleague in the classroom often feels as though it depends on stereotypes associated with ethnicity.

"It's hard for exchange students [...] Just having an accent makes people not want to have them in their groups [because they think] it'll be harder for them to get good marks for presentations and stuff."

Female students often identified experiencing or witnessing sexism on campus as another major environmental stressor. Several female students discussed feeling the need to work harder, and be more active in all areas of academia (e.g., academics, extra curriculars, etc.) than male students in order to be acknowledged. Female students often expressed concerns surrounding their ability to be successful in the "boys club" of academia and other professional settings, with one student sharing a jarring interaction she had with a male supervisor:

\section{"He turned to me and said, 'Women are} bringing down this specialty because they keep leaving to have children.",

In addition to feeling the pressures associated with sexism, female students also reported experiencing instances of sexual harassment on campus, with some noting that they avoid certain places and times of day on campus due to feeling unsafe.
Competitive Atmosphere. One of the most common stressors discussed during consultations was competition between peers. Almost all students remarked on the competitive atmosphere on campus, and the need to perform at a competitive level at all times and "be better than the person sitting next to them." One student shared:

[We're] trying to continuously climb up this ladder, and a lot of people don't know when to stop and end up stretching themselves too thin. [...] You're always feeling like your peers are smarter than you, [so] you try to overachieve constantly.

This sentiment of imposter syndrome was common, particularly among graduate students. Many students reported feeling as though their peers were smarter or more successful than them, feeling as though they were never working hard enough, or feeling like they didn't deserve the accolades they had been given.

"People are like, "You're doing great! You're exactly where you need to be. You're going to make a great scientist!" And I'm like ... I've tricked them! Am I a good actor? [...] I feel like someone at some point is going to call my bluff, and I'm just constantly waiting for that shoe to drop."

Students in professional programs shared similar feelings, in addition to emphasizing the demand to constantly be successful without showing the strain it places on the individual. One student noted the importance of finding a positive coping strategy to manage the level of stress you're expected to withstand:

"[A big part of our success] is how good we get at either becoming numb to it, or convincing ourselves we're good enough, or actually feeling 
like we're good enough... if you don't come up with a coping strategy you're screwed."

Some students spoke about the benefits of the competitive atmosphere, including being consistently pushed to perform at the highest level and learn as much as possible. However, the pressure does come with its drawbacks:

"[The stress] does light a fire under you, to push you to be better [...] But it really does test peoples' resilience. If you're regularly being convinced that you're insufficient, if you don't have enough self-confidence... at some point you'll run out."

Adjustment to Post-Secondary. Adjusting to the post-secondary setting and its associated expectations was also identified as a source of stress, particularly for incoming undergraduate students. Graduate students reflected back on their experience, often noting in hindsight just how challenging the initial transition had been. One student shared:

"I think they need to make undergrads very aware of what's coming [...] your grades are going to drop, you are going to feel incredibly overwhelmed, you need to be prepared for this because it will happen. I went from honour roll in high school to academic probation. You're going to be a little bit miserable."

Beyond the academic transition, students emphasized adjustments in all areas of life, from becoming responsible for managing your own time and schedule, to managing new interactions in the social setting.

\section{Interpersonal Stressors}

Stressors in the interpersonal realm addressed the difficulties with cultivating and maintaining friendships and relationships with peers and family members. Students also touched on the stress associated with meeting others' expectations, learning to live with roommates, and comparing themselves to others on social media.

Relationship Management. Students shared the challenges they experienced with both forming new friendships, and maintaining old ones upon beginning their post-secondary career. Several students argued that it was relatively easy to make acquaintances, but much more difficult to form genuine friendships. On her first-year experience, one student shared:

"My closest friends [from high school] went to a different university and they all lived together. I wanted to maintain those relationships, but while I was doing that it made it hard to meet new people at my own school."

At the other end of the spectrum, graduate students often remarked on the challenges associated with maintaining friendships with those outside of academia. One student shared:

"Most of my friends are working professionals now, so it's stressful to balance my school work with their schedules, because their work doesn't spill over into their personal life. As a student, you're never really 'off.,",

In a similar vein, several participants discussed the stress associated with maintaining a relationship with their parents, noting that often, family members fail to understand the amount of responsibilities students manage on a day-to-day basis. Graduate students echoed these comments, adding that family members often attempt to poke fun at the length of time they have spent in the academic world. While this teasing is no doubt meant to be good-natured, students emphasized the stress this produces, flaring feelings of inadequacy and invalidating their academic and professional goals. One student shared: 
"My Dad says my career is being a "professional student" [...] it's belittling. There seems to be a stigma around being a student. People label you as immature because you're still in school as opposed to [working in] the 'real world.",

In a final note on relationships, many students emphasized the stress they sometimes experience as a result of roommates or housemates. While students shared the positives as well ("It's a lot of fun... [but] not a lot of work gets done"), they emphasized the challenges with late nights, social tension, and living conditions. On the topic of sleep, one student shared:

"When you're in high school living at home with your family, when you want to sleep you can really sleep. When you're living with housemates, people are always moving around and doing something, staying up late... you wake up in the middle of the night all the time."

Meeting Expectations. Students also discussed stress associated with meeting the expectations they set for themselves, as well as the expectations of others. Students identified selfexpectations such as feeling that they weren't as involved as they should be, or that they weren't working hard enough. One student shared:

"I'm involved in two nationwide studies, I am a teaching assistant, and I have a part time job. I'm tired all the time [...] my schedule is very, very full, but I always feel like I'm not doing enough. [...] I'm constantly comparing myself to other people."

Students also discussed expectations placed on them as a result of their program of study. For example, one student shared the constant pressures associated with studying medicine with respect to academic, professional, and personal success, noting the unrealistic expectations for medical students to excel in every domain ("We are all expected to be leaders in every area").

Similarly, a commerce student emphasized the impact one's reputation can have on their future opportunities within the faculty, as well as future opportunities, and the pressure this can place on an individual:

"It's so easy to get caught up in the undercurrent of all the pressure and expectations and what people think of you [...] People love to talk... especially about people that they know."

Many students emphasized struggling to meet the expectations placed on them others, including family, friends, and in some cases, society in general. Students emphasized that meeting the expectations of family members was especially stressful if family was contributing to a student's education financially. One student shared:

"Your parents have these high expectations for you and they are spending so much money for you [to be here], and they want you to achieve all these wonderful milestones. [If] you don't achieve them, you feel like you're letting everybody down."

Another common subtheme that emerged was the stress caused by the advent of social media. Students cited the newfound pressures that perfectly curated "online selves" can place on students. One student shared their feelings, highlighting that despite knowing that what is presented online is often not an honest reflection of reality, social media still manages to spark feelings of competition and inadequacy:

"What I see [people] post about on Instagram, Facebook, Snapchat [...] that's not really what they're going through. They are probably going through very similar things [to me], and they're 
seeing me in a better light too. [...] You just

have that desire to compare yourself to

somebody else and it's so tough."

\section{Personal}

Stressors within the personal realm were wideranging, addressing concerns about one's selfcare and health, work/life balance, financial stress, concern for the future. The sources of stress outlined in this domain were largely linked to the overarching challenge of time management.

Self-Care and Health. The most commonly identified stressors within the personal domain related to self-care and managing one's health. Students identified challenges like getting enough sleep, getting enough exercise, and eating a nutritious diet while trying to balance the other demands in their academic, professional and social lives. One student expressed the following:

"There is this culture that expects us to sacrifice our sleep, our meal times... sacrifice these things that are not extras - not luxuries - but required to function as a human being. We're supposed to sacrifice these things to do well in school [...] There's this weird, disgusting badge of honour that comes along with being the "best" at sacrificing. Like, 'I only got four hours of sleep, 'well, 'I didn't sleep at all','

This sentiment regarding a "culture of sacrifice" was reiterated by many students, with many noting that managing a very full schedule necessitates having to let something slide, with these simple elements of self-care often the first to go. On attempting to work in time to exercise, one student shared:

"Sometimes I have so much stuff to do, and I'm just so exhausted that I don't want to go to the gym - that's the last thing I want to do. [...] I have so many things that are more important to do right now than spending half an hour on an elliptical."

Work/Life Balance. On a similar note to sacrificing self-care activities, many students identified struggling to find a work/life balance as a major source of stress. Students emphasized the difficulty with balancing academic responsibilities, with extracurriculars or jobs outside of school, in addition to attempting to find time for hobbies and interests. One student noted:

"There is such a push on this extremely unhealthy work/life balance and I think it leads to a lot of burnout. [...] It's the perfect storm for mental health problems."

Often, students identified a feeling of guilt associated with taking any time for relaxation, or engagement with hobbies or interests outside of academia. This sentiment was particularly evident among graduate and professional students, one of whom shared:

"[Taking time for] my hobbies is stress relieving in the moment, but the minute I stop doing it, I'm like "Oh my God, that's ten minutes I could've been working on this other thing, what was I thinking?"

Financial Stressors. Concerns surrounding finances were also commonly emphasized by students as a significant source of stress. Unsurprisingly, students who had taken student loans described significant stress associated with their incurred debt, particularly those students who were nearing graduation. Students noted that money (and in particular, debt) was always in the back of their mind, sometimes promoting uncertainty about continuing on with one's studies. One graduate student shared: 
"When I'm done, I'm going to have 60 or $70 \mathrm{k}$ dollars of debt that I'll never be able to pay back. [...] I often wonder... is it even worth doing this PhD to become that much more in debt?"

Other students noted that their lack of financial literacy caused them day-to-day stress. Students remarked on the stress associated with not only the expenses associated with academics (e.g., tuition, books, student fees), but also those dayto-day expenses including rent, food, and utilities. One student summed up their feelings concisely: "Money is stressful because I don't understand it."

Concern for the Future. Worrying about "next steps" following the completion of their postsecondary education was a prevalent stressor noted among both undergraduate and graduate students. Students shared significant concerns about finding a career following graduation, with many linking their financial stressors with their perceived prospects in the job market. One student shared their experience, having been the first in their family to attend post-secondary education and achieve an undergraduate degree:

"I just spent \$40,000 working my ass off... I just spent all this money, all this time doing this, and everyone was like, "You have a degree, that's so awesome!" And then, I'm applying for jobs and they're like, "Oh, you only have an undergrad... we don't want you" [...] I was working the same minimum wage job I worked before [going to school], and it felt like it was all for nothing."

While medical students shared stressors associated with matching to a residency program, undergraduate students aspiring to complete graduate education expressed fears over not being accepted in their program of choice. Regardless of area of study and future aspirations (e.g., work place or program of study), all students expressed a fear of the uncertainty of their futures. A prevalent concern, particularly for students in long-term programs (e.g., medical school, or graduate-level education), including the uncertainty around reaching major life events such as finding a partner, getting married, and/or starting a family. One doctoral student shared:

"My friends are all getting married and having children. Even though I know I'm achieving a personal goal to get the career I want [...] I'm so far behind on those important life events."

This was a particularly common concern among female students, regardless of program of study, with many women voicing concerns around managing timing for starting a career versus starting a family, and feeling as though choosing one would mean failing at the other.

\section{Discussion}

In this study, students from a variety of levels and areas of study at an Ontario university were engaged in discussions surrounding the sources of post-secondary student stress, and the social context within which students live and learn. Five major themes of stress were derived from the data, including: academics, the learning environment, campus culture, interpersonal, and personal stressors. Findings revealed key areas for potential improvement on post-secondary campuses across Canada regarding the promotion of student mental health and wellness.

When considering post-secondary student stress, and particularly stress related to academics, often stressors such as test anxiety and the pressure to maintain high grades spring to the forefront of conversations (Cairns, Massfeller, \& Deeth, 2010; Gerwing, Rash, Allen Gerwing, Bramble, \& Landine, 2015; Kruisselbrink Flatt, 2013; Robinson, Jubenville, Renny, \& Cairns, 2016; Ruthig, Haynes, Stupnisky, \& Perry, 2009). While these stressors continue be prevalent 
among post-secondary students, remarks shared by students in this study indicated that the stress surrounding these routine challenges is perhaps more multifaceted. The subtext of time management was prevalent throughout discussions surrounding stressors in both the academic domain and others, suggesting that campuses should perhaps adjust their approach from teaching students how to study for and complete assignments, tests, and examinations, and shift focus towards developing multitasking and time management skill building programs that are applicable not only to academics, but to all areas of life (e.g., relationship management, etc.).

In keeping with the time management theme, students expressed a common, skewed perception of the requirements for being a "successful" student, and to maintain that success following graduation. Data revealed that students felt that keeping an overly full schedule, with little to no "down" time for things like self-care and socialization was required of them, and expressed a tendency to intentionally take on more than was manageable in order to check all the boxes to meet expectations for "success." Fear of failure was evident among students in this sample, and has been well-documented in the literature, as well as linked to negative mental health outcomes (Monk, 2004; Villatte, Marcotte, \& Potvin, 2017). Institutions should work towards normalizing expectations for students regarding failure and work to emphasize the importance of self-care and recharging as a valuable tool for true "success."

The importance of positive and productive staffstudent relationships should also by highlighted as a key area of concern for post-secondary institutions moving forward. While the importance of the supervisory relationship on graduate students' positive academic outcomes and sense of belonging in academia has been well-documented (Curtin, Stewart, \& Ostrove, 2013; Devos et al., 2017; Jairam \& Kahl, 2012), the literature is less clear with respect to the impact of poor teaching on students' academic and mental health outcomes at the post-secondary level. Perhaps most concerningly, students emphasized reluctance to seek academic accommodations, both informal (e.g., asking for a one-time extension) and formal (e.g., longstanding academic accommodations), noting that doing so seemed to reflect an inability to meet expectations and was often met with stigma (Lindsay, Cagliostro, \& Carafa, 2018; Thompson-Ebanks \& Jarman, 2018) and the unwillingness or implied dissatisfaction of faculty, something recently noted in the literature (Kendall, 2016; Lindsay et al., 2018). This is particularly concerning, given the recent increase in conversations surrounding the roles and responsibilities of faculty with respect to recognizing changes in, and supporting students' mental health and wellbeing (DiPlacitoDeRango, 2018). Mental health literacy and education for staff and faculty should be prioritized alongside student wellness.

Consistent with previous evidence suggesting that social integration into the campus culture and social space is a key component of students' ability to thrive (Brook \& Willoughby, 2015), data from this study placed emphasis on the importance of promoting a positive campus culture. In this study, socio-cultural stressors largely hinged on unhealthy competitiveness, again driving feelings of failure and overburdened students, as well as experiences of discrimination within the post-secondary setting, including racism and sexism. Experiences of discrimination have been widely recorded in the literature, with students belonging to ethnic minorities consistently reporting higher stress levels and poorer mental health than their counterparts (Hawley et al., 2016), and experiences of racism and sexism linked to the 
prediction of depression among undergraduate students in the United States (Dinh, Holmberg, Ho, \& Haynes, 2013). Institutions should continue to work towards cultivating a positive campus culture of inclusiveness and diversity, where students feel safe and respected. The Okanagan Charter, now adopted by several postsecondary institutions across Canada, presents a useful guiding framework in this area (Okanagan Charter, 2015).

\section{Limitations}

While a variety of students were involved in this study in terms of both level, year, and area of study, some limitations remain. We were unable to recruit international students, a subgroup that has been routinely identified as being at risk for isolation and the development of mental healthrelated problems. Focus groups were small, ranging from 2-5 participants in each, which may have resulted in a smaller breadth of data and idea sharing than may have been garnered from larger groups. Conversely, it is possible that the small group size promoted more intimate conversation and disclosure regarding sensitive topics. Finally, while diverse, students were recruited from a single university, and therefore some of the themes that emerged may be specific to the postsecondary institution, rather than representative of those experienced by students across Canada. Finally, at the time of this research, the principle investigator (BL) was also a post-secondary student who shared many similar experiences of stress with participants. It is therefore likely that the interpretation of this data was biased by the investigator's personal experiences.

\section{Conclusions}

The purpose of this study was to generate conversations with post-secondary students about sources of stress within the post-secondary setting, and gain insight into the social context surrounding these stressors. The goal of this study was to invite students to identify salient sources of stress within the post-secondary setting in an attempt to better evaluate these stressors moving forward. This research has resulted in the identification of five major themes of stress among Canadian post-secondary students, including: academics, the learning environment, campus culture, interpersonal, and personal stressors. Themes and sub-themes revealed in this research echo those previously identified in a major scoping review of the academic and grey literature, conducted in support of the development of Canada's National Standard for the Psychological Health and Safety of PostSecondary Students (Linden, Gray, \& Stuart, 2018). The findings from this research have been used to develop a new instrument for evaluating post-secondary student stress (Linden \& Stuart, 2019), and can assist institutions with refining and improving the targeting of upstream student health services including mental health promotion and mental illness prevention programming.

\section{Acknowledgements}

The authors would like to acknowledge the participants for sharing their personal experiences and contributing towards the betterment of post-secondary mental health and wellbeing. The authors would also like to thank LTS for initial input and review of this manuscript.

\section{Declaration of Interest}

The authors declare that they have no potential conflicts of interest to disclose.

\section{Funding}

This research was conducted as a component of BL's doctoral dissertation, and as such, was financially supported by [blinded] University's Department of Public Health Sciences and the Health Services and Policy Research Institute.

\section{Notes on Contributors}

Brooke Linden, PhD is a Postdoctoral Research Fellow in the Health Services and Policy Research Institute at Queen's university in Kingston, Canada. 
Heather Stuart, $\mathbf{P h D}$ is a Professor in the Department of Public Health Sciences at Queen's University in Kingston, Canada, and the Bell Canada Mental Health and Anti-Stigma Research Chair.

\section{References}

American College Health Association. (2016). American College Health Association - National College Health Assessment II: Canadian Reference Group Data Report Spring 2016. Retrieved from http://www.acha-ncha.org/

American College Health Association. (2019). American College Health Association - National College Health Assessment II: Canadian Reference Group Data Report Spring 2019. Retrieved from www.acha.org

Arnett, J. J. (2000). Emerging Adulthood: a theory of development from the late teens through the twenties. American Psychologist, 55(5), 469480. doi: 10.1037/0003-066X.55.5.469

Bradshaw, C., Atkinson, S., \& Doody, O. (2017). Employing a Qualitative Description Approach in Health Care Research. Global Qualitative Nursing Research, 4. doi: $10.1177 / 2333393617742282$

Brook, C. A., \& Willoughby, T. (2015). The social ties that bind: social anxiety and academic achievement across the university years. Journal of Youth and Adolescence, 44(5), 1139-1152. doi: 10.1007/s10964-015-0262-8

Cairns, S. L., Massfeller, H. F., \& Deeth, S. C. (2010). Why do postsecondary students seek counselling? Canadian Journal of Counselling, 44(1), 34-50.

Curtin, N., Stewart, A., \& Ostrove, J. (2013). Fostering academic self-concept: advisor support and sense of belonging among international and graduate students. American Educational Research Journal, 50(1), 108-137. doi: 10.3102/0002831212446662

Devos, C., Boudrenghien, G., Van der Linden, N., Azzi, A., Frenay, M., Galand, B., \& Klein, O. (2017). Doctoral students' experiences leading to completion or attrition: a matter of sense, progress and distress. European Journal of Psychology of Education, 32(1), 61-77. doi: 10.1007/s10212-016-0290-0
Dinh, K. T., Holmberg, M. D., Ho, I. K., \& Haynes, M. C. (2013). The relationship of prejudicial attitudes to psychological, social, and physical well-being within a sample of college students in the United States. Journal of Cultural Diversity, 21(2), 56-66.

DiPlacito-DeRango, M. L. (2018). Situating the postsecondary instructor in a supportive role for the mental health and well-being of students. International Journal of Mental Health and Addiction, 16(2), 284-290. doi: 10.1007/s11469-017-9740-4

Eisenberg, D., Gollust, S. E., Golberstein, E., \& Hefner, J. L. (2007). Prevalence and correlates of depression, anxiety, and suicidality among university students. American Journal of Orthopsychiatry, 77(4), 534-542. doi: 10.1037/0002-9432.77.4.534

Gerwing, T. G., Rash, J. A., Allen Gerwing, A. M., Bramble, B., \& Landine, J. (2015). Perceptions and Incidence of Test Anxiety. The Canadian Journal for the Scholarship of Teaching and Learning, 6(3), 12-23.

Gollust, S. E., Eisenberg, D., \& Golberstein, E. (2008). Prevalence and correlates of self-injury among university students. Journal of American College Health, 56(5), 491-498. doi: 10.3200/JACH.56.5.491-498

Guest, G., MacQueen, K., \& Namey, E. (2012). Applied Thematic Analysis. California: SAGE Publications, Inc.

Hawley, L. D., MacDonald, M. G., Wallace, E. H., Smith, J., Wummel, B., \& Wren, P. A. (2016). Baseline assessment of campus-wide general health status and mental health: opportunity for tailored suicide prevention and mental health awareness programming. Journal of American College Health, 64(3), 174-183. doi: 10.1080/07448481.2015.1085059

Jairam, D., \& Kahl, D. (2012). Navigating the doctoral experience: the role of social support in successful degree completion. International Journal of Doctoral Studies, 7, 311-330. doi: $10.28945 / 1700$

Kendall, L. (2016). Higher education and disability: exploring student experiences. Cogent Education, $\quad 3(1), \quad 1-12 . \quad$ doi: 10.1080/2331186X.2016.1256142 
Kruisselbrink Flatt, A. (2013). A suffering generation: six factors contributing to the mental health crisis in North American higher education. College Quarterly, 16(1), 17.

Linden, B., Gray, S., \& Stuart, H. (2018). National standard for the psychological health and safety of post-secondary students - Phase I: Scoping literature review. Retrieved from https://www.mentalhealthcommission.ca/sites/d efault/files/2018-

10/Scoping_Review_Post_Secondary_Student Mental_Health_eng.pdf

Linden, B., \& Stuart, H. (2019). Psychometric assessment of the Post- Secondary Student Stressors Index (PSSI). BMC Public Health, 19(1139), 1-12. doi: 10.1186/s12889-019-7472$\mathrm{Z}$

Lindsay, S., Cagliostro, E., \& Carafa, G. (2018). A systematic review of barriers and facilitators of disability disclosure and accommodations for youth in post-secondary education. International Journal of Disability, Development and Education, 65(5), 1-31. doi: 10.1080/1034912X.2018.1430352

Monk, E. (2004). Student mental health: the case studies. Counselling Psychology Quarterly, 17(4), 395-412. doi: $10.1080 / 09515070412331331200$

Navaneelan, T. (2012). Suicide rates: an overview. doi: Statistics Canada Catalogue no. 82-624-X

Okanagan Charter. (2015). Okanagan Charter: An international charter for health promoting universities and colleges. Retrieved from https://wellbeing.ubc.ca/okanagan-charter

Patel, V., Flisher, A. J., Hetrick, S., \& McGorry, P. (2007). Mental health of young people: a global public-health challenge. The Lancet, 369(9569), 1302-1313. doi: 10.1016/S01406736(07)60368-7

Pearson, C., Janz, T., \& Ali, J. (2013). Mental and substance use disorders in Canada (Catalogue no. 82-624-X). doi: Statistics Canada Catalogue no. 82-624-X

Robinson, A. M., Jubenville, T. M., Renny, K., \& Cairns, S. L. (2016). Academic and mental health needs of students on a Canadian campus. Canadian Journal of Counselling and Psychotherapy, 50(2), 108-123.
Ruthig, J., Haynes, T., Stupnisky, R., \& Perry, R. (2009). Perceived Academic Control: mediating the effects of optimism and social support on college students' psychological health. Social Psychology of Education, 12(2), 233-249. doi: 10.1007/s11218-008-9079-6

Statistics Canada. (2017). Education in Canada: Key results from the 2016 census. 1-13. doi: Statistics Canada Catalogue no. 11-001-X

Sunderland, A., \& Findlay, L. C. (2013). Perceived need for mental health care in Canada: results from the 2012 Canadian Community Health Survey-Mental Health. Health Reports, 24(9), 3-9. doi: Catalogue no. 82-003-X

Thompson-Ebanks, V., \& Jarman, M. (2018). Undergraduate students with nonapparent disabilities identify factors that contribute to disclosure decisions. International Journal of Disability, Development and Education, 65(3), 286-303. doi: 10.1080/1034912X.2017.1380174

Villatte, A., Marcotte, D., \& Potvin, A. (2017). Correlates of depression in first-year college students. The Canadian Journal of Higher Education, 47(1), 114-136.

Walsh, G. (2018). The Cost of Credentials: The shifting burden of post-secondary tuition in Canada. Retrieved from http://www.rbc.com/economics/economicreports/pdf/other-reports/Tuition_June2018.pdf 